\title{
Successful Certolizumab Pegol Treatment of Chronic Anterior Uveitis Associated with Psoriasis Vulgaris
}

\author{
Keiko Yamaguchi ${ }^{\mathrm{a}}$ Takaaki Hayashi $^{\mathrm{a}}$ Genichiro Takahashi ${ }^{\mathrm{a}}$ \\ Mami Momose $^{b} \quad$ Akihiko Asahina $^{\mathrm{b}}$ Tadashi Nakano $^{c}$ \\ aDepartment of Ophthalmology, Katsushika Medical Center, The Jikei University School of \\ Medicine, Tokyo, Japan; 'bepartment of Dermatology, The Jikei University School of \\ Medicine, Tokyo, Japan; 'Department of Ophthalmology, The Jikei University School of \\ Medicine, Tokyo, Japan
}

\section{Keywords}

Certolizumab pegol treatment · Chronic anterior uveitis · Psoriasis vulgaris

\section{Abstract}

This report presents details on a 45-year-old male Japanese patient with chronic and refractory anterior uveitis associated with psoriasis vulgaris who was administered certolizumab pegol (CZP), which is an anti-tumor necrosis factor alpha (TNF- $\alpha$ ) monoclonal antibody. Although CZP has only been formally approved for rheumatoid arthritis treatment in Japan, a clinical trial allowed us to assess CZP effectiveness in this patient. The grade 3+ anterior chamber inflammation (for both the cells and flare) observed at baseline improved to grade 0 at 3 months post-treatment. Dermatologically, the psoriasis area severity index (PASI) score was 25.4 , while the body surface area (BSA) was $88 \%$ at baseline. At 3 months after treatment, the scores improved to 2.8 for PASI and less than $1 \%$ for BSA. After the treatment, remission has lasted for at least 9 months. No adverse events were seen during the CZP treatment. These findings suggest that CZP could be an effective therapeutic alternative in some refractory anterior uveitis patients with psoriasis vulgaris. 


\section{Introduction}

Psoriasis is a chronic, relapsing, inflammatory autoimmune skin disease. Uveitis commonly occurs in some patients with psoriasis $[1,2]$. Typical uveitis associated with psoriasis is regarded as acute non-granulomatous iridocyclitis. Although it has been thought that posterior segment inflammation rarely occurs, several recent studies have reported finding posterior segment lesions [3-5]. Furthermore, about half of the patients become affected bilaterally several years after the original onset [6]. Tumor necrosis factor alpha (TNF- $\alpha$ ) is an important inflammatory cytokine that is involved in various inflammatory diseases, including rheumatoid arthritis (RA), Crohn's disease, Behçet's disease, and psoriasis. Nickoloff [7] demonstrated that the important factors in the immunopathogenesis of psoriasis included TNF- $\alpha$ and interleukin (IL)-23, among others. Therefore, in addition to conventional therapy, biologics such as anti-TNF- $\alpha$ antibodies (infliximab [IFX], adalimumab [ADA], and etanercept) and anti-IL-12/23p40 monoclonal antibody (ustekinumab) have been considered as alternative treatment options. Certolizumab pegol (CZP) is a PEGylated Fab' fragment of an anti-TNF$\alpha$ monoclonal antibody that can bind to both membrane-bound and soluble TNF- $\alpha$. However, in Japan, CZP has only been approved for the treatment of RA. Here, we report a patient in whom psoriasis vulgaris and chronic uveitis were successfully treated with CZP.

\section{Case Report}

A Japanese male was clinically diagnosed with psoriasis vulgaris at age 24 . He had a previous medical history of femur head necrosis at age 23 . At age 41 , his psoriasis vulgaris diagnosis was pathologically confirmed by a skin biopsy performed at a previous hospital where oral immunosuppressive drugs were administered but failed due to adverse events. Subsequently, the patient was followed up using oral anti-allergic drugs, active vitamin D3 ointment, and topical steroid eye drops. At age 42, he visited the Katsushika Medical Center at Jikei University due to left eye pain. A blood test showed that while he was homozygously positive for HLA-A2, he was negative for HLA-B27. Active iridocyclitis and posterior iris adhesions were seen in both eyes. Fundoscopy revealed no remarkable findings. Although topical betamethasone phosphate and tropicamide/phenylephrine hydrochloride were administered for the active anterior uveitis, his inflammatory control was poor due to the occurrence of uveitis that was accompanied by hypopyon at least once. Thereafter, his ocular symptoms were gradually relieved through the use of oral anti-allergic, active vitamin D3 ointment, and topical betamethasone phosphate eye drops. It was not possible to use any of the available approved antiTNF- $\alpha$ agents IFX and ADA for the psoriasis vulgaris or noninfectious uveitis at the time of the original treatment due to the patient's financial situation. As a result, we decided to include this patient in a clinical trial for the use of CZP (Cimzia ${ }^{\circledR}$; UCB, Inc., Smyrna, GA, USA) for the treatment of psoriasis vulgaris at the Department of Dermatology of Jikei University Hospital. Baseline examination of his decimal best-corrected visual acuity (BCVA) at age 45 was 1.2 and $1.5 \mathrm{in} \mathrm{his} \mathrm{right} \mathrm{and} \mathrm{left} \mathrm{eye,} \mathrm{respectively.} \mathrm{His} \mathrm{intraocular} \mathrm{pressure} \mathrm{was} 11$ and $7 \mathrm{~mm} \mathrm{Hg}$ in his right and left eye, respectively. Based on the standard method for grading anterior chamber cells [8], the anterior chamber inflammation grade was $3+$ for cells and $3+$ for flare in each eye (Fig. 1a, b). Posterior synechiae and posterior subcapsular cataracts were noted in both eyes (Fig. 1a, b). Fundoscopy and spectral-domain optical coherence tomography (Cirrus HD OCT; Carl Zeiss Meditec AG, Dublin, CA, USA) revealed no remarkable findings (Fig. 1c, d). OCT imaging of his left eye could not be performed due to advanced posterior iris adhesion. 
Assessment of the dermatological disease severity showed the psoriasis area severity index (PASI) score was 25.4, while the body surface area (BSA) was $88 \%$. The patient did not have psoriatic arthritis. The level of C-reactive protein (CRP) was $10.75 \mathrm{mg} / \mathrm{dL}$. Topical $0.1 \%$ fluorometholone was administered 3 times per day bilaterally. CZP was administered by subcutaneous injection every 2 weeks. The 3 initial doses administered were $400 \mathrm{mg}$, followed by $200 \mathrm{mg}$ at 2 -week intervals. After 1 month, the treatment was successful with the anterior chamber inflammation grade improving to $0.5+$ cells without flare in each eye. After 3 months of treatment, BCVA was 1.2 and 0.8 in his right and left eye, respectively. Decreased vision in the left eye was due to progressive posterior subcapsular cataract. The anterior chamber inflammation disappeared in both eyes (grade: 0 ). Dermatological findings markedly improved to 2.8 for the PASI and to less than $1 \%$ for the BSA scores. The CRP value decreased to 0.73 $\mathrm{mg} / \mathrm{dL}$. At 9 months after the start of the CZP, there was no anterior chamber inflammation observed, and the use of topical $0.1 \%$ fluorometholone was discontinued. The CRP value was $0.40 \mathrm{mg} / \mathrm{dL}$. At the last follow-up visit at 12 months after the CZP treatment, no anterior chamber inflammation was observed. No adverse events were observed during the CZP treatment.

\section{Discussion}

In Japan, only the anti-TNF- $\alpha$ agents IFX and ADA are available for the treatment of psoriasis vulgaris or refractory noninfectious uveitis. This is the first report of a successful treatment with CZP in a Japanese patient with chronic anterior uveitis associated with psoriasis vulgaris.

Previous studies have shown that patients with uveitis associated with psoriasis usually exhibit acute non-granulomatous anterior uveitis, which is consistent with the findings for our current patient [6]. A recent large-scale study demonstrated that a group of patients with severe psoriasis who received systemic therapy had an increased risk of uveitis compared to patients with mild psoriasis who did not receive any systemic therapy [2]. In psoriasis-associated uveitis patients, it is very important to treat not only the ocular inflammation but also the psoriasis vulgaris [6]. Huynh et al. [9] examined the use of biologic agents in patients with psoriatic ocular inflammatory disease and reported that 7 out of 8 patients treated with IFX or ADA achieved remission of their ocular inflammation. The effectiveness of IFX or ADA has also been reported in Japanese patients with psoriatic uveitis $[5,6]$.

CZP has been recently approved for use in treating inflammatory diseases, including RA. Results of an inflammatory arthritis study showed that not only was the penetration of CZP into arthritic murine paws greater as compared with IFX and ADA, but there was also a longer duration of drug exposure in the inflamed tissues [10]. These features of CZP may be attributable to the PEGylation and the smaller molecular weight, as compared with IFX and ADA. A recent study also additionally showed that CZP monotherapy was an effective and well-tolerated therapeutic alternative for the treatment of psoriasis and psoriatic arthritis, although this study did not discuss the ophthalmic complications [11]. Llorenç et al. [12] examined 7 patients with refractory uveitis, 2 of whom had psoriatic arthritis. All 7 of these patients initially received IFX, after which they were switched to ADA due to the development of serious adverse events [12]. In all 7 patients, CZP was subsequently administered at the same dose as was used in our current patient, with CZP shown to be an effective treatment in refractory uveitis [12]. However, CZP treatment has yet to be performed in uveitis patients with psoriasis vulgaris in Japan, as CZP has only been formally approved for use in treating RA. After the 
administration of CZP for psoriasis vulgaris in our current patient, this led to a complete remission of the chronic anterior uveitis after the treatment.

Patients with psoriatic uveitis usually exhibit refractory ocular inflammation. Furthermore, a recurrence of uveitis was observed in $69 \%$ of psoriatic uveitis patients [6]. Similarly, it was also very difficult to achieve remission of the chronic anterior uveitis in our current patient when just using topical betamethasone phosphate, with remission only achieved after starting the CZP treatment. The successful results that were obtained after using the new biological agent CZP,are encouraging for ophthalmologists, as well as dermatologists. However, further studies will need to be undertaken in order to confirm the efficacy and safety of CZP for uveitis related to psoriasis vulgaris.

In conclusion, the complete remission response observed in our patient suggests that CZP could be an effective therapeutic alternative for some refractory uveitis patients with psoriasis vulgaris.

\section{Statement of Ethics}

The use of CZP was approved by the Institutional Review Board at The Jikei University School of Medicine. Written informed consent was obtained from the patient regarding the use of CZP.

\section{Disclosure Statement}

A.A. has received a research grant from UCB Japan Co., Ltd.

\section{References}

1 Catsarou-Catsari A, Katsambas A, Theodoropoulos P, Stratigos J. Ophthalmological manifestations in patients with psoriasis. Acta Derm Venereol. 1984;64(6):557-9.

2 Chi CC, Tung TH, Wang J, Lin YS, Chen YF, Hsu TK, et al. Risk of Uveitis Among People With Psoriasis: A Nationwide Cohort Study. JAMA Ophthalmol. 2017 May;135(5):415-22.

3 Paiva ES, Macaluso DC, Edwards A, Rosenbaum JT. Characterisation of uveitis in patients with psoriatic arthritis. Ann Rheum Dis. 2000 Jan;59(1):67-70.

4 Durrani K, Foster CS. Psoriatic uveitis: a distinct clinical entity? Am J Ophthalmol. 2005 Jan;139(1):106-11.

5 Sakurai Y, Namba K, Mizuuchi K, Nomura T, Ishida S. A Case of Severe Panuveitis Associated with Psoriasis Vulgaris Successfully Treated with Infliximab. Case Rep Ophthalmol. 2016 Apr;7(1):191-4.

6 Tanaka R, Takamoto M, Komae K, Ohtomo K, Fujino Y, Kaburaki T. Clinical features of psoriatic uveitis in Japanese patients. Graefes Arch Clin Exp Ophthalmol. 2015 Jul;253(7):1175-80.

7 Nickoloff BJ. Cracking the cytokine code in psoriasis. Nat Med. 2007 Mar;13(3):242-4.

8 Jabs DA, Nussenblatt RB, Rosenbaum JT; Standardization of Uveitis Nomenclature (SUN) Working Group. Standardization of uveitis nomenclature for reporting clinical data. Results of the First International Workshop. Am J Ophthalmol. 2005 Sep;140(3):509-16.

9 Huynh N, Cervantes-Castaneda RA, Bhat P, Gallagher MJ, Foster CS. Biologic response modifier therapy for psoriatic ocular inflammatory disease. Ocul Immunol Inflamm. 2008 May-Jun;16(3):89-93.

10 Chimenti MS, Saraceno R, Chiricozzi A, Giunta A, Chimenti S, Perricone R. Profile of certolizumab and its potential in the treatment of psoriatic arthritis. Drug Des Devel Ther. 2013 Apr;7:339-48.

11 Dattola A, Cannizzaro MV, Mazzeo M, Bianchi L. Certolizumab Pegol in the Treatment of Psoriasis and Psoriatic Arthritis: Preliminary Real-Life Data. Dermatol Ther (Heidelb). 2017 Dec;7(4):485-92.

12 Llorenç V, Mesquida M, Sainz de la Maza M, Blanco R, Calvo V, Maíz O, et al. Certolizumab Pegol, a New AntiTNF- $\alpha$ in the Armamentarium against Ocular Inflammation. Ocul Immunol Inflamm. 2016;24(2):167-72. 


\section{Case Reports in Ophthalmology}
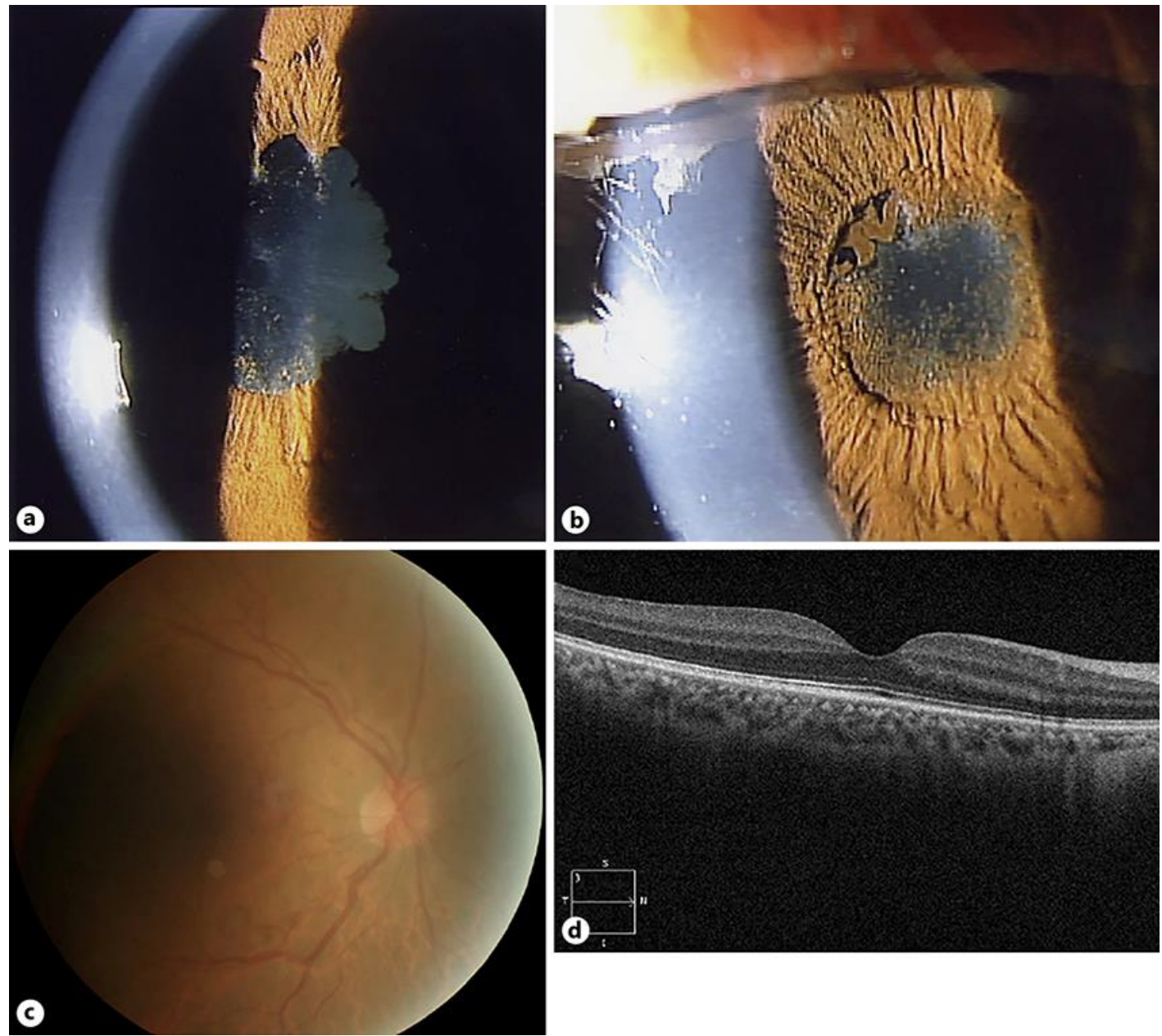

Fig. 1. Slit lamp images of the anterior segment with mydriasis in the right (a) and left (b) eyes, showing anterior chamber inflammation (grade: $3+$ for cells and 3+ for flare) and posterior synechiae in each eye. A fundus photograph (c) and spectral-domain optical coherence tomography (d) reveal no remarkable findings in the right eye. 